\title{
Managing cluster headache with sphenopalatine ganglion stimulation: a review
}

\author{
Denys Fontaine ${ }^{1,2}$ \\ Serena Santuccil,2 \\ Michel Lanteri-Minet ${ }^{2-4}$ \\ 'Department of Neurosurgery, $\mathrm{CHU}$ \\ de Nice, Université Cote d'Azur, \\ Nice, France; ${ }^{2}$ Université Cote d'Azur, \\ FHU INOVPAIN, CHU de Nice, Nice, \\ France; ${ }^{3}$ INSERM/UdA, Auvergne \\ University, Clermont-Ferrand, France; \\ ${ }^{4}$ Pain Department, CHU de Nice, \\ Université Cote d'Azur, Nice, France
}

Correspondence: Denys Fontaine Service de Neurochirurgie, Hopital Pasteur, CHU de Nice, 30 Avenue de la Voie Romaine, 06000 Nice, France Tel +33492038450

Fax +33492038425

Email fontaine.d@chu-nice.fr
This article was published in the following Dove Press journal: Journal of Pain Research

\begin{abstract}
Cluster headache $(\mathrm{CH})$ is a primary headache and considered as one of the worst pains known to man. The sphenopalatine ganglion (SPG) plays a pivotal role in cranial autonomic symptoms associated with pain. Lesioning procedures involving the SPG and experimental acute SPG stimulation have shown some degree of efficacy with regard to $\mathrm{CH}$. A neuromodulation device, chronically implanted in the pterygopalatine fossa, has been specifically designed for acute on-demand SPG stimulation. In a pilot placebo-controlled study in 28 patients suffering from refractory chronic $\mathrm{CH}$, alleviation of pain was achieved in $67.1 \%$ of full stimulation-treated attacks compared to $7 \%$ of sham stimulation-treated attacks ( $p<0.0001)$. Long-term results ( 24 months; 33 patients) confirmed the efficacy of SPG stimulation as an abortive treatment for $\mathrm{CH}$ attacks. Moreover, $35 \%$ of the patients observed a $>50 \%$ reduction in attack frequency, suggesting that repeated use of SPG stimulation might act as a $\mathrm{CH}$-preventive treatment. Globally, $61 \%$ of the patients were acute responders, frequency responders, or both, and 39\% did not respond to SPG stimulation. The safety of SPG microstimulator implantation procedure was evaluated in a cohort of 99 patients; facial sensory disturbances were observed in $67 \%$ of the patients $(46 \%$ of them being transient), transient allodynia in $3 \%$, and infection in 5\%. SPG stimulation appears as a promising innovative, efficient, and safe therapeutic solution for patients suffering from severe $\mathrm{CH}$. It has shown its efficacy in aborting $\mathrm{CH}$ attacks compared to placebo stimulation, suggesting that it is particularly adapted for $\mathrm{CH}$ patients who are not sufficiently improved by abortive treatments such as sumatriptan and oxygen. However, further studies comparing SPG stimulation with standard abortive and/or preventive $\mathrm{CH}$ treatments will be necessary to define more precisely its place within the management of severe chronic and/or episodic $\mathrm{CH}$.
\end{abstract}

Keywords: cluster headache, primary headache, sphenopalatine ganglion, stimulation, neuromodulation

\section{Cluster headache}

Cluster headache $(\mathrm{CH})$ is a primary headache and is the most common form of trigeminal autonomic cephalalgias that are grouped in the third section of the International Headache Society Classification (ICHD-III). ${ }^{1} \mathrm{CH}$ is considered to be one of the worst pains known to man. Individuals often describe the pain like having a "red hot poker" penetrating one eye. They report that the intensity is so extreme it is unlike anything they have ever experienced, and female patients describe each attack as being worse than childbirth. The intensity of pain is so high that it can induce suicidal thoughts, and so $\mathrm{CH}$ has been named as "suicidal headache". Such severity justifies greater attention to this disease.

$\mathrm{CH}$ is characterized by recurrent attacks of very severe unilateral pain, usually located in and around the orbit. During an attack, ipsilateral cranial autonomic 
symptoms (lacrimation, conjunctival injection, nasal congestion or rhinorrhea, ptosis, edema of the eyelid or the face, sweating of the forehead or the face, miosis) accompany the pain, and behavior is characterized by a marked sense of agitation and necessity to move. Attacks generally last 15 minutes to 3 hours and occur from once every day to eight times daily, sometimes with a striking circadian rhythmicity, with some patients reporting a predictability of onset during the day or, more frequently, during the night, waking from sleep. The term $\mathrm{CH}$ is related to the tendency of attacks to cluster together into bouts that last several weeks or months. In the episodic $\mathrm{CH}$, the bouts occur at certain times of year and are separated by periods of remission, which last at least a month. Frequently, episodic $\mathrm{CH}$ has a striking circannual periodicity, with the bouts occurring in the same month of the year. However, about $10 \%-20 \%$ of individuals have the chronic form of $\mathrm{CH}$ and have continuous attacks with no respite or remission periods lasting less than 1 month. ${ }^{1}$

While there is currently no cure for $\mathrm{CH}$, the available treatment has become more effective in the last 20 years, especially the acute treatments. ${ }^{2}$ Acute treatment is used to stop the pain once it has started, and the key element is speed to reduce the excruciating pain as fast as possible. For this purpose, conventional analgesia is ineffective, and only two treatments are validated, oxygen inhalation and subcutaneous sumatriptan. Preventive and transitional treatment, attempting to reduce frequency and intensity of attacks, is based on verapamil, lithium, or corticosteroids. Due to failures of these treatments in refractory patients, neuromodulation approaches have been introduced in the management of $\mathrm{CH}$ during the last 10 years. Invasive methods, namely deep brain stimulation (DBS), occipital nerve stimulation (ONS), and sphenopalatine ganglion (SPG) stimulation are reserved for patients with severe and refractory $\mathrm{CH}$, but emergent noninvasive methods might serve as useful adjuncts to more conventional therapies.

\section{CH physiopathology and SPG involvement}

The pathophysiology of $\mathrm{CH}$ has not been completely identified yet. The individual $\mathrm{CH}$ attack is regarded as an activation of the trigemino-autonomic reflex, and the posterior hypothalamus is supposed to play a pivotal role, probably via a trigemino-hypothalamic pathway. ${ }^{3}$ This pathophysiological model explains the three major features of $\mathrm{CH}$ : unilateral trigeminal distribution of pain, ipsilateral cranial autonomic symptoms, and circadian episodic pattern of attacks. ${ }^{4}$ Nevertheless, many issues still remain unanswered. Few neuroimaging studies have explored $\mathrm{CH}$ patients, ${ }^{5}$ suggesting structural $^{6}$ and functional ${ }^{7}$ changes in a posterior hypothalamic region. Finally, a genetic component is suggested by the increased familial risk (14-fold and twofold increased risk in first- and second-degree relatives, respectively). ${ }^{8}$

The SPG is believed to play a pivotal role in cranial autonomic symptoms associated with pain and might be involved in headache pain. Recent hypothesis suggests that, during $\mathrm{CH}$ attacks, there is an activation of the parasympathetic superior salivatory nucleus and SPG parasympathetic fibers, inducing neuropeptides release (especially calcitonin gene-related peptide) and vasodilatation of the cerebral and dural blood vessels, which activate meningeal nociceptive fibers projecting to the trigeminal ganglion and nuclei. These processes induce referred pain in the periorbital region. Thus, blockage of the SPG, by any means, might theoretically interfere with this pathological process and treat $\mathrm{CH}$ attack.

The SPG contains sensory fibers innervating the posterior nasopharynx, sympathetic fibers from the superior cervical ganglion via the vidian nerve, and preganglionic parasympathetic fibers coming from the superior salivatory nucleus via the greater petrosal nerve and connecting, within the SPG, with postganglionic fibers. The SPG is located in the pterygopalatine fossa (PPF), behind the posterior wall of the maxillary sinus (Figure 1), bordered posteriorly by the pterygoid process, superiorly by the sphenoid sinus, and medially by the palatine bone. Laterally, the PPF communicates with the

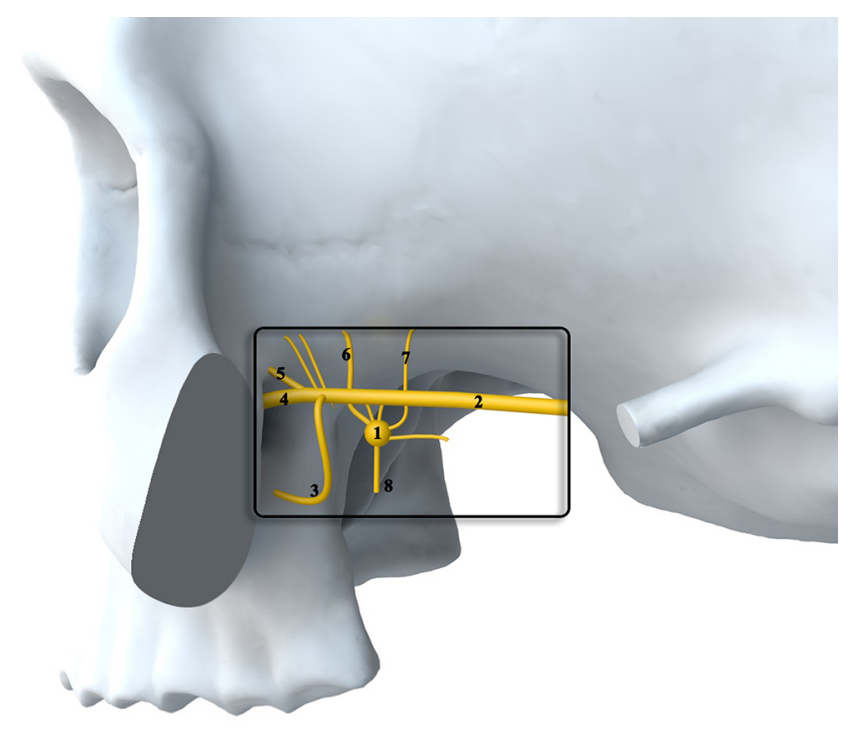

Figure I Schematic and magnified representation of the SPG anatomy within the PPF.

Notes: I: Sphenopalatine (pterygopalatine) ganglion. 2: Maxillary division of the trigeminal nerve (V2). 3: Posterior superior alveolar nerve. 4: Infra-orbital nerve. 5: Zygomatic nerve. 6: Nasal nerves. 7: Pharyngeal nerves. 8: Palatine nerves. Abbreviations: PPF, pterygopalatine fossa; SPG, sphenopalatine ganglion. 
infratemporal fossa. The maxillary division of the trigeminal nerve (V2) passes the foramen rotundum located superolaterally within the PPF, and is in close contact with the SPG.

\section{SPG as a therapeutic target for $\mathbf{C H}$}

For more than a century, SPG has been targeted to treat primary headache, either by surgical ganglionectomy, ${ }^{9}$ lidocaine application, ${ }^{10}$ percutaneous lesioning, ${ }^{11}$ and, more recently, electrical stimulation.

Several lesioning procedures involving the SPG have been proposed for the treatment of chronic $\mathrm{CH}$. The success rates of ganglionectomy, radiofrequency lesion, or blocks varied from $46 \%$ to $85 \% .^{9,11-13}$ However, repeated access to the SPG was often necessary because, in most of the cases, the benefits were transient. Efficacy of SPG radiofrequency lesioning, using a percutaneous infra-zygomatic approach, has been studied in two series, cumulating 25 patients (mean follow-up 12-24 months). ${ }^{11,13}$ The mean frequency of chronic $\mathrm{CH}(\mathrm{CCH})$ attacks was decreased by half after the procedure. However, the complication rate was high and included such complications as epistaxis $(80 \%)$, lesion of the maxillary division of the trigeminal nerve (40\%), and transient hypoesthesia of the palatine area $(90 \%)$.

Stereotactic radiosurgery (SRS) of the SPG had been proposed initially to treat $\mathrm{CH}$ in combination with trigeminal nerve SRS and seemed slightly more efficient than trigeminal nerve SRS alone. ${ }^{14}$ SRS targeting only the SPG has been reported to be efficient in single cases. ${ }^{15,16}$

Globally destructive procedures on the SPG appeared to be partially efficient on $\mathrm{CCH}$, which has encouraged the development of new nonlesional procedures targeting the SPG.

\section{Electrical stimulation of the SPG}

Based on the effect of lesioning procedures on the SPG, its stimulation was proposed for the treatment of $\mathrm{CH}$ by Ibarra for the first time in 2007, using an implantable device in a single case. ${ }^{17}$ The author mentioned a marked reduction of pain with acceptable safety. The only complication reported was hardware failure, after which the patient's headaches worsened. Interestingly, his condition improved after replacement of the stimulator, suggesting the efficacy of SPG stimulation. In 2010, Ansarinia showed that acute SPG electrical stimulation was able to abolish provoked $\mathrm{CH}$ attack within few minutes in six patients suffering from refractory chronic $\mathrm{CH}^{18}{ }^{18}$ In this study, SPG stimulation was delivered using a transient electrode, implanted percutaneous by an infra-zygomatic approach.

Based on these preliminary results, a chronically implantable neuromodulation device ("Pulsante"), specifically designed for acute SPG stimulation, has been developed by the company Autonomic Technologies (ATI, Redwood City, CA, USA), in order to abort the $\mathrm{CH}$ attacks on-demand (Figure 2). The neurostimulator device is implanted in the PPF, along the posterior wall of the maxillary bone, and fixed to the zygomatic process with a screwed plate, with the lead being placed in contact with the SPG (Figure 3). The neurostimulator does not contain a battery but is activated and powered by a remote controller using radiofrequency energy.

To evaluate the safety and efficacy of SPG stimulation, we conducted a Medline search using the terms "sphenopalatine ganglion", "stimulation", and "cluster headache", excluding case reports, technical reports, review articles, and redundant series. Only two articles, originating from the same multicentric group of physicians, were finally considered. ${ }^{19,20}$

The safety and efficacy of this SPG stimulation device have been assessed in a pilot multicenter study (Pathway CH-1 study), in 28 patients suffering from refractory $\mathrm{CCH}^{20}$ with at least four attacks per week. In this sham-controlled study, each $\mathrm{CH}$ attack was randomly treated with 15-minute full stimulation, subperception stimulation, or sham stimulation

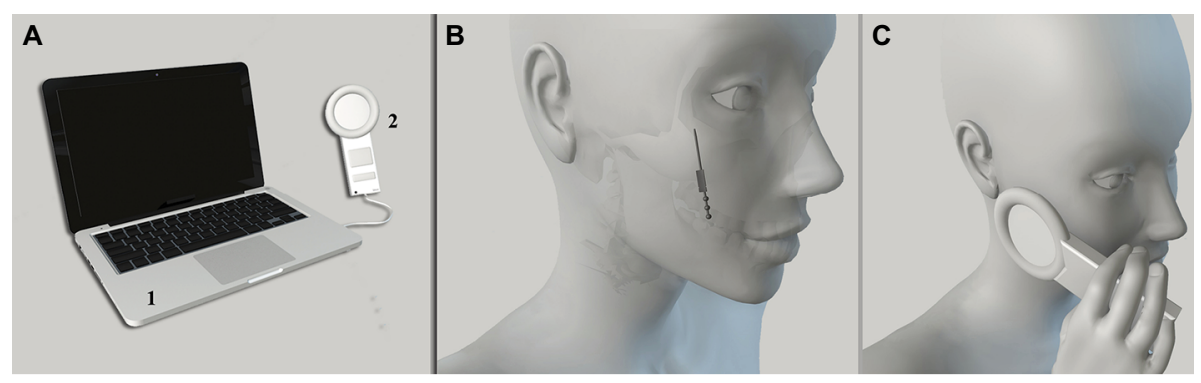

Figure 2 Principle of the Pulsante system (Autonomic Technologies, Redwood City, CA, USA) designed for acute on-demand stimulation of the SPG.

Notes: (A) External programming hardware. The program software (I) allows the physician to test and program the neurostimulator parameters to customize stimulation for each patient. The remote controller (2) is a rechargeable handheld device used by the patient to activate the neurostimulator, and it is also used by the physician to communicate with the neurostimulator during programming sessions. (B) The miniaturized neurostimulator is implanted in the PPF to stimulate the SPG and is fixed by screws to the maxillary bone. (C) Activation of the stimulation by the patient to abort the $\mathrm{CH}$ attack by application of the remote controller against the patient's cheek.

Abbreviations: $\mathrm{CH}$, cluster headache; PPF, pterygopalatine fossa; SPG, sphenopalatine ganglion. 


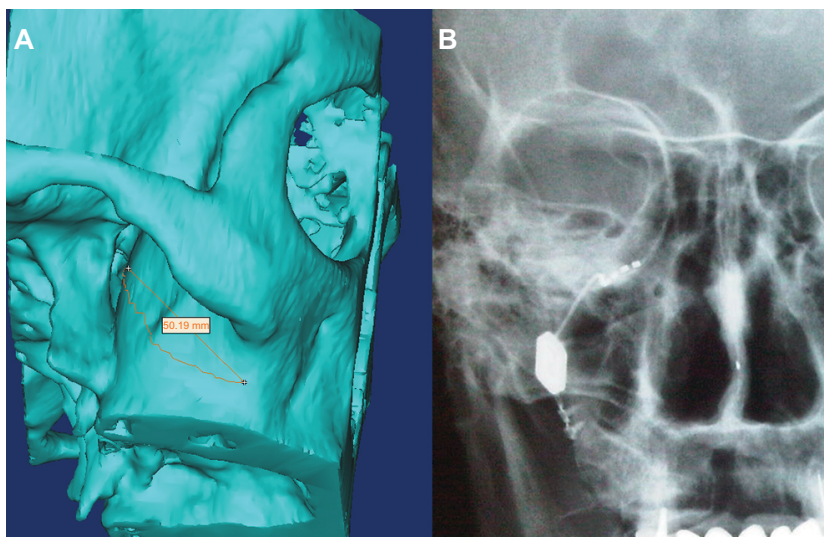

Figure 3 Pre- and postoperative imaging.

Notes: (A) Preoperative CT scan tridimensional reconstruction allowing to plan the implantation of the SPG stimulation device within the PPF. (B) Postoperative anteroposterior craniofacial radiograph showing the placement of the SPG stimulation device.

Abbreviation: CT, computed tomography; PPF, pterygopalatine fossa; SPG, sphenopalatine ganglion.

(last two stimulation modes being nonactive). Absence of pain was defined as reduction of pain intensity by 2 points or more according to a Categorical Pain Scale to 0 points, and alleviation of pain to 0 or 1 point(s). Alleviation of pain was achieved in $67.1 \%$ of full stimulation-treated attacks compared to $7.4 \%$ of sham-treated and $7.3 \%$ of subperceptiontreated attacks $(p<0.0001)$. Absence of pain was achieved in $34.1 \%$ and $1.5 \%$ of attacks after active stimulation and sham stimulation, respectively $(p<0.0001)$. For $\mathrm{CH}$ attacks treated with full stimulation, pain relief was achieved in $55.5 \%$ and $60.6 \%$, at 30 and 60 minutes, respectively. Nineteen of 28 (68\%) patients experienced a clinically significant improvement, but only $32 \%$ achieved a pain relief in more than $50 \%$ of the treated attacks. Moreover, $43 \%$ of the patients experienced a reduction $>50 \%$ of attack frequency (average $88 \%$ ).

Long-term (24 months) results of the open phase of this study have been recently published, ${ }^{19}$ in 33 patients. All patients were initially included in the Pathway CH-1 study, although 11 of them were not included in the first analysis (for time reasons). Moreover, 10 patients from the initial study were excluded from this long-term analysis, because they no longer had the stimulator implanted or due to previous protocol noncompliance. Across all 33 patients, a total of 5,956 attacks were treated. Effective treatment (alleviation and/or absence of pain) was achieved in $65 \%$ of $\mathrm{CH}$ attacks, with a delay of 11.2 minutes on average, including $50 \%$ becoming pain free. Fifteen out of 33 patients (45\%) were considered as acute responders (at least $50 \%$ of attacks were successfully treated). In $79 \%$ of the attacks, patients did not report the use of acute medication. Moreover, 11 patients out of 31 (35\%) observed a $>50 \%$ reduction of the weekly attack frequency 24 months after implantation compared with the 4 -week period before implantation (average reduction was $83 \%$ ). These data suggested that repeated use of the SPG stimulation might act as a $\mathrm{CH}$ prophylactic treatment, although spontaneous transformation from chronic to episodic forms of the disease cannot be excluded. Globally, $61 \%$ of the patients were acute responders, frequency responders, or both, and $39 \%$ of the patients did not respond to SPG stimulation.

Headache disability (Headache Impact Test [HIT-6 ${ }^{\mathrm{TM}}$ ] scale) was significantly improved (decrease $>2.3$ points) in $55 \%$ of the patients, and 21 patients out of $33(64 \%)$ were able to decrease or stop ( 9 cases) their prophylactic $\mathrm{CH}$ medication.

\section{Complications and side effects}

In the pilot Pathway-CH1 study, ${ }^{20} 81 \%$ of the patients experienced transient, mild-to-moderate hypoesthesia within the maxillary (V2) nerve territory, resolving within 3 months in most of the cases. More recently, the safety of the surgical implantation procedure of the Pulsante SPG stimulator has been evaluated in a cohort of 99 patients, including 43 patients of the Pathway $\mathrm{CH}-1$ study and 56 patients from the Pathway-R1 registry. ${ }^{21}$ Eighty one percent of the patients experienced at least one adverse event, most of them being transient. Sensory disturbances were the most frequent complications, observed in $67 \%$ of the patients, $46 \%$ of them resolving within a mean delay of 104 days. Transient allodynia was rare (3\%). Pain and/or swelling were reported by $47 \%$ of the patients, resolving in $80 \%$ of the cases with a mean delay of 68 days. Dry eye (3\%, resolving in $40 \%$ of cases), transient trismus (8\%), and limited jaw movements $(6 \%)$ were also reported.

Infection rate was $5 \%$. Microstimulator revision procedures were performed in 13 cases due to inappropriate initial 
placement of the stimulating electrode within the PPF. Five stimulators were explanted.

Although frequent, most (92\%) of the adverse events were transient and evaluated as mild or moderate. The authors concluded that SPG microstimulator insertion procedure has sequelae comparable to other oral cavity procedures including tooth extractions, sinus surgery, and dental implant placement. Moreover, the technique is recent, and the rate of surgical complications will likely decrease with progression of the learning curve, further refinement of the surgical procedure and tools, and the use of neuronavigation systems. ${ }^{22}$

\section{Limitations}

Although SPG stimulation appears as a promising, safe, and efficient technique to treat $\mathrm{CH}$ attacks, data assessing its safety and efficacy remain limited, and the quality of evidence concerning this therapy, according to the GRADE assessment system, ${ }^{23}$ is low. All the currently available data come from a single study and a registry, including a limited number of patients (respectively, 43 and 56 patients), and both of these studies were promoted and supported by the company commercializing the implantable device. In the pilot study, ${ }^{20} 15$ out of the 28 reported patients have had less than $10 \mathrm{CH}$ attacks during the randomized phase, meaning that the conclusions were based mostly on the analysis of the remaining 13 patients. This study compared active stimulation with sham stimulation and demonstrated that SPG stimulation therapeutic effect was not due to a placebo effect. However, no study compared SPG stimulation with the current standard abortive treatment, namely sumatriptan injection and/or oxygen inhalation, in terms of efficacy, delay between treatment administration and pain relief, amplitude of pain relief, respective constraints and safety, and costs. Further studies will have to address these points. Similarly, although a preventive effect has been suggested by the association of repeated SPG stimulation and decrease of attack frequency, no study compared SPG stimulation with $\mathrm{CH}$ prophylactic treatment or other surgical alternatives for refractory chronic $\mathrm{CH}$, namely ONS and DBS.

These data will be needed to determine the exact place of SPG stimulation in the management of $\mathrm{CH}$. Moreover, all the previous data have been collected in refractory $\mathrm{CH}$, and no published data are available regarding the interest of SPG stimulation in episodic $\mathrm{CH}$.

\section{Place of SPG stimulation in the management of $\mathrm{CH}$}

Despite these limitations, SPG stimulation is a promising innovative, efficient, and safe therapeutic solution for patients in severely disabling painful condition. As an acute treatment to abort attacks, this procedure is particularly adapted for patients who do not respond to current abortive treatment as sumatriptan and oxygen. In a recent study on refractory $\mathrm{CCH}$ patient candidates for ONS, ${ }^{24} 19 \%$ of them did not use subcutaneous sumatriptan or oxygen because these treatments were ineffective, not tolerated, or contraindicated. These patients, experiencing $20 \mathrm{CH}$ attacks per week on average, would benefit dramatically from an alternative abortive treatment such as SPG stimulation. About $30 \%$ of refractory $\mathrm{CCH}$ attacks do not respond to ONS. ${ }^{24}$ Considering their respective risks, we consider that ONS and SPG stimulation should be proposed before DBS in medically intractable $\mathrm{CCH}$ patients. However, the choice between ONS and SPG stimulation as a first surgical treatment is difficult and should be based on benefits/risks ratio, patient's anatomy and headache characteristics, local availability of the different techniques, regulatory and reimbursement issues, and national guidelines.

Clinical criteria for invasive surgery, initially DBS, in $\mathrm{CCH}$ have been proposed by a group of experts as follows: ${ }^{25}$ $\mathrm{CCH}$ for at least 2 years, at least one attack per day, resistance to pharmacotherapy, headache "locked" to the same side, normal neurological examination, and absence of psychiatric comorbidity. Selection criteria for SPG stimulation may be moderated and/or extended, considering that SPG stimulation is less invasive and risky than DBS and acts as an abortive treatment. An expert consensus proposed in 2014 selection criteria for SPG stimulation to be as follows: 1) CCH according to ICHD classification;,26 2) documented for at least 2 years; 3 ) refractory to medical treatment, ${ }^{27}$ which is defined as failed adequate trials of preventive medication, alone or in combination (failure being defined as no therapeutic or unsatisfactory effect, intolerable side effects, or contraindications to use; adequate being defined as appropriate dose and appropriate length of time); 4) with substantial number of weekly attacks; 5) severe psychosocial impairment; and 6) attacks being side locked or occurring predominantly $(>90 \%)$ on the current side for the last 12 months. Patients must be able to understand the device use and ideally be able to document their attacks on a diary. Implantation should be performed in a center with expertise in headache diagnosis and treatment, and having a surgical team with specific training and expertise in SPG stimulator implantation.

No recommendation was given concerning episodic $\mathrm{CH}$, as the clinical data currently published have been obtained in $\mathrm{CCH}$. However, patients with episodic CH (ICHD-III classification) for at least 2 years, with active periods lasting several months, 
and having several daily attacks not or poorly responding to abortive treatments might benefit from SPG stimulation.

Exclusion criteria for SPG stimulation have been identified as follows: previous history of destructive procedure on the SPG ganglion; severe psychiatric disorder (other than reactive depression); particular anatomical conditions as narrow lateral opening of the PPF (evaluated on preoperative computed tomography scan); recent ( $<4$ months) facial surgery in the PPF area or zygomatico-maxillary buttress; active regional infectious process (periodontal disease, open carious lesions, osteomyelitis, abscess, etc); previous ( $<6$ months) facial malignancy or radiotherapy; and craniofacial disease requiring frequent magnetic resonance imaging (Pulsante system seems compatible with $1.5 \mathrm{~T}$ magnetic resonance imaging but generates image artifacts) or surgery in the next months.

In a position statement on neuromodulation techniques in chronic headaches published in 2013, a group of experts from the European Headache Federation considered that, given that only one placebo-controlled study was available, SPG stimulation had to be seen as experimental until further studies are presented. ${ }^{28}$ This opinion was shared by the guidelines for the treatment of $\mathrm{CH}$ published by the French Headache Society in 2014, ${ }^{29}$ who recommended, in addition, to reserve this therapy to tertiary headache centers. Pulsante system has obtained a CE mark (Conformité Européene [European Conformity]) for the treatment of $\mathrm{CH}$ but is not yet approved by the US Food and Drug Administration, and is not yet reimbursed by all the health care providers.

\section{Conclusion}

SPG stimulation appears as a promising innovative, efficient, and safe therapeutic solution for patients suffering from severe CH. SPG stimulation has showed its efficacy to abort $\mathrm{CH}$ attacks versus placebo stimulation, suggesting that it is particularly adapted for $\mathrm{CH}$ patients who are not sufficiently improved by currently available abortive treatments such as sumatriptan and oxygen. Additionally, the pilot study suggested that repeated SPG stimulation might have a preventive action on $\mathrm{CH}$ attack frequency. However, further studies comparing SPG stimulation with standard abortive and/or preventive $\mathrm{CH}$ treatments will be necessary to define more precisely its place within the management of severe chronic and/or episodic $\mathrm{CH}$.

\section{Disclosure}

D Fontaine and M Lanteri-Minet are consultants for Autonomic Technologies and Medtronic. The authors report no other conflicts of interest in this work.

\section{References}

1. Headache Classification Committee of the International Headache Society. The International Classification of Headache Disorders: 3rd edition beta version. Cephalalgia. 2013;33(9):629-808.

2. Robbins M, Starling A, Pringsheim T, Becker W, Schwedt T. Treatment of cluster headache: the American Headache Society evidence-based guidelines. Headache. 2006;56(7):1093-1106.

3. Goadsby P. Pathophysiology of cluster headache: a trigeminal autonomic cephalalgia. Lancet Neurol. 2002;1:251-257.

4. May A. Cluster headache: pathogenesis, diagnosis and management. Lancet Neurol. 2005;366(9488):843-855.

5. Tso A, Goadsby P. Recent neuroimaging advances in the study of primary headache. Curr Pain Headache Rep. 2015;19(6):15.

6. May A, Ashburner J, Buchel C, et al. Correlation between structural and functional changes in brain in an idiopathic headache syndrome. Nat Med. 1999;5(7):836-838.

7. May A, Bahra A, Buchel C, Frackowiak R, Goadsby P. Hypothalamic activation in cluster headache attacks. Lancet Neurol. 1998;352(9124): 275-278.

8. Russel M. Epidemiology and genetics of cluster headache. Lancet Neurol 2004;3:279-283.

9. Meyer J, Binns P, Ericsson A, Vulpe M. Sphenopalatine gangionectomy for cluster headache. Arch Otolaryngol. 1970;92(5):475-484.

10. Costa A, Pucci E, Antonaci F, et al. The effect of intranasal cocaine and lidocaine on nitroglycerin-induced attacks in cluster headache. Cephalalgia. 2000;20(2):85-91.

11. Narouze S, Kapural L, Casanova J, Mekhail N. Sphenopalatine ganglion radiofrequency ablation for the management of chronic cluster headache. Headache. 2009;49(4):571-577.

12. Devoghel J. Cluster headache and sphenopalatine block. Acta Anaesthesiol Belg. 1981;32(1):101-107.

13. Sanders M, Zuurmond W. Efficacy of sphenopalatine ganglion blockade in 66 patients suffering from cluster headache: a 12- to 70-month follow-up evaluation. J Neurosurg. 1997;87(6):876-880.

14. Kano H, Kondziolka D, Mathieu D, et al. Stereotactic radiosurgery for intractable cluster headache: an initial report from the North American Gamma Knife Consortium. J Neurosurg. 2011;114(6):1736-1743.

15. De Salles A, Gorgulho A, Golish S, et al. Technical and anatomical aspects of Novalis stereotactic radiosurgery sphenopalatine ganglionectomy. Int J Radiat Oncol Biol Phys. 2006;66(Suppl 4): S53-S57.

16. Lad S, Lipani J, Gibbs I, Chang S, Adler J, Henderson J. Cyberknife targeting the pterygopalatine ganglion for the treatment of chronic cluster headaches. Neurosurg. 2007;60(3):E580-E581.

17. Ibarra E. Neuromodulacion del Ganglio Esfenopalatino para Aliviar los Sintomas de la Cefalea en Racimos. Reporte de un Caso. Boletin El Dolor. 2007;64(16):12-18.

18. Ansarinia M, Rezai A, Tepper S, et al. Electrical stimulation of sphenopalatine ganglion for acute treatment of cluster headaches. Headache. 2010;50(7):1164-1174.

19. Jurgens T, Barloese M, May A, et al. Long-term effectiveness of sphenopalatine ganglion stimulation for cluster headache. Cephalagia. 2017;37(5):423-434.

20. Schoenen J, Jensen R, Lantéri-Minet M, et al. Stimulation of the sphenopalatine ganglion (SPG) for cluster headache treatment. Pathway CH-1: a randomized, sham-controlled study. Cephalalgia. 2013;33(10):816-830.

21. Assaf A, Hillerup S, Rostgaard J, et al. Technical and surgical aspects of the sphenopalatine ganglion (SPG) microstimulator insertion procedure. Int J Oral Maxillofac Surg. 2016;45(2):245-254.

22. Kohlmeier C, Behrens $P$, Böger A, et al. Improved surgical procedure using intraoperative navigation for the implantation of the SPG microstimulator in patients with chronic cluster headache. Int J Comput Assist Radiol Surg. 2017;12(12):2119-2128.

23. Guyatt G, Oxman A, Schunemann H, Tugwell P, Knottnerus A. GRADE guidelines: A new series of articles in the Journal of Clinical Epidemiology. J Clin Epidemiol. 2011;64(4):380-382. 
24. Fontaine D, Blond S, Lucas C, et al. Occipital nerve stimulation improves the quality of life in medically-intractable chronic cluster headache: results of an observational prospective study. Cephalalgia. 2017;37(12): 1173-1179.

25. Leone M, May A, Franzini A, et al. Deep brain stimulation for intractable chronic cluster headache: proposals for patient selection. Cephalalgia. 2004;24(11):934-937.

26. Headache Classification Committee of the International Headache Society. The International Classification of Headache Disorders: 2nd edition. Cephalalgia. 2004;24(Suppl 1):9-160.
27. Goadsby P, Schoenen J, Ferrari M, Silberstein S, Dodick D. Towards a definition of intractable headache for use in clinical practice and trials. Cephalalgia. 2006;26(9):1168-1170.

28. Martelletti P, Antal A, Arcioni R, et al. Neuromodulation of chronic headaches: position statement from the European Headache Federation. J Headache Pain. 2013;14(1):86.

29. Donnet A, Demarquay G, Ducros A, et al. French guidelines for the management of cluster headache (French Headache Society). Rev Neurol (Paris). 2014;170(11):653-670.
Journal of Pain Research

\section{Publish your work in this journal}

The Journal of Pain Research is an international, peer reviewed, open access, online journal that welcomes laboratory and clinical findings in the fields of pain research and the prevention and management of pain. Original research, reviews, symposium reports, hypothesis formation and commentaries are all considered for publication.

\section{Dovepress}

The manuscript management system is completely online and includes a very quick and fair peer-review system, which is all easy to use. Visit http://www.dovepress.com/testimonials.php to read real quotes from published authors. 